\title{
Evaluación de simuladores como estrategia para el aprendizaje de la electricidad en la asignatura de física en la educación media
}

\section{Evaluation of simulators as a strategy for the learning of electricity in the subject of physics in middle education.}

Pablo Luis Rodriguez Abril ${ }^{1}$

Ariel Adolfo Rodriguez-Hernández ${ }^{2}$

Fanny Avella-Forero ${ }^{3}$

Grupo de Investigación TICA - Universidad

Pedagógica y Tecnológica de Colombia

\section{RESUMEN}

Este trabajo de investigación identifico y evalúo los simuladores gráficos computacionales que

1 pabloluis.rodriguez@uptc.edu.co

https://orcid.org/0000-0003-0493-791X

Grupo Investigación TICA. Universidad Pedagógica y Tecnológica de Colombia

$2 \quad$ ariel.rodriguez@uptc.edu.co

https://orcid.org/0000-0003-1906-7734

Grupo de Investigación TICA / Tecno Innovaciones /

TelemaTICs - Universidad Pedagógica y Tecnológica de Colombia

$3 \quad$ Fanny.avella@uptc.edu.co

https://orcid.org/0000-0002-4207-1777

Grupo de Investigación TICA - Universidad Pedagógica y

Tecnológica de Colombia pueden ser utilizados como parte del diseño de unaestrategiapedagógica que integresu uso para la apropiación de conceptos de electrodinámica en estudiantes de grado once. Como aporte a la solución de problemática generada por la enseñanza descontextualizada de la física eléctrica que dificulta que el estudiante resuelva problemas usando la ciencia, baja su interés por la asignatura, baja gradualmente los resultados en pruebas externas, y retarda los avances en la dinámica social de los municipios. Por lo que se hace necesario usar en el aula herramientas de calidad inviertan esta tendencia. Para la 
evaluación de los simuladores se usó la guía para el análisis descriptivo de la funcionalidad del software de simulación propuesta por Ayala y Salinas (2019), la cual propone evaluar software tipo simulador bajo ocho aspectos significativos (identificación, funcionalidad, confiabilidad, usabilidad, eficiencia, mantenimiento y portabilidad y propuesta didáctica). Se realizó la revisión de simuladores y se logró identificar dos simuladores PHET y Crocodile que por sus características presentan un mayor grado de adaptabilidad al contexto, al cumplimiento de los objetivos de aprendizaje y competencias a desarrollar dentro del proceso de aprendizaje de la electrodinámica en la educación media.

\section{PALABRAS CLAVE:}

simuladores gráficos computacionales, evaluación de simuladores educativos, simulación en la educación.

\section{ABSTRACT}

This research work identified and evaluated computational graphic simulators that can be used as part of the design of a pedagogical strategy that integrates their use for the appropriation of electrodynamic concepts in eleventh grade students. As a contribution to the solution of problems generated by the decontextualized teaching of electrical physics that makes it difficult for the student to solve problems using science, lowers their interest in the subject, gradually lowers the results in external tests, and delays progress in social dynamics of the municipalities. Therefore, it is necessary to use quality tools in the classroom to reverse this trend. For the evaluation of the simulators, the guide for the descriptive analysis of the functionality of the simulation software proposed by Ayala and Salinas (2019) was used, which proposes to evaluate simulatortype software under eight significant aspects (identification, functionality, reliability, usability, efficiency, maintenance and portability and didactic proposal). The simulator review was carried out and it was possible to identify two PHET and Crocodile simulators that, due to their characteristics, present a greater degree of adaptability to the context, to the fulfillment of the learning objectives and skills to be developed within the learning process of electrodynamics in education.

\section{KEY WORDS:}

computer graphics simulators, evaluation of educational simulators, simulation in Education.

\section{INTRODUCCIÓN}

Teniendo en cuenta el rápido avance de la sociedad en temas de desarrollo, economía y tecnología, surge la necesidad de brindar formación de calidad en ciencias que propenda por favorecer procesos de participación activa en la sociedad por medio de la solución de problemas (Nieda \& Macedo, 1997). La UNESCO plantea la relevancia de enseñar las ciencias como la física, química para garantizar el derecho a la educación e incentivar la formación de ciudadanos activos e informados por medio de capacidades científicas endógenas de la población (UNESCO, 1999).

Tomando como referente a Ostermann \& Moreira (2020) se explica como por medio de la enseñanza de las ciencias se promueve el cambio de los contextos para llevar a cabo un proceso educativo de calidad. De igual forma, se evidencia que la enseñanza de las ciencias en grados superiores es considerada difícil y abstracta por la mayoría de loes estudiantes lo que dificulta su proceso escolar. Además, Parra et al. (2015) identifican la dificultad que se presenta en la aplicación de estrategias de enseñanza de las ciencias debido a la falta de recursos de las instituciones educativas para brindar ambientes de aprendizaje adecuados que incluyan laboratorios de mediana complejidad. 
Los resultados de las pruebas saber, en área de ciencias, aplicadas entre los años 2016 y 2019 a estudiantes de secundaria de la Institución Educativa Enrique Suárez del municipio de Almeida en Boyacá, reflejan que, en cuanto a las competencias del uso comprensivo del conocimiento científico, explicación de fenómenos e indagación y los conceptos del componente físico, los estudiantes presentan un promedio de error superior al compararlos con los resultados departamentales y nacionales. De igual manera, se constata que el porcentaje de error se eleva con cada año que transcurre. Los porcentajes de error elevados traen como consecuencia un bajo desempeño y la disminución de la calidad del aprendizaje de las ciencias.

Por las anteriores razones surge el proyecto de investigación en curso: "Aprendizaje de conceptos en física eléctrica integrando simuladores." que plantea la pregunta: “¿Cómo la integración de una propuesta pedagógica utilizando simuladores en el aula influye en la apropiación de conceptos en física eléctrica en estudiantes de grado once de la institución educativa Enrique Suarez del municipio de Almeida?" y pretende: integrar una propuesta pedagógica con el uso simuladores en el aula, para la apropiación de los conceptos de física eléctrica en estudiantes de grado once de la Institución Educativa Enrique Suarez de Almeida, Boyacá.

Algunas de las soluciones a la problemática expuesta contemplan el uso de simuladores graficos computaciones ( e.g. Martinez, 2016). Debido a que el proyecto está en curso, la presente ponencia expone el proceso que se lleva a cabo para lograr la selección de los simuladores gráficos computacionales idóneos que permitan abordar la problemática y propenda por mejorar las condiciones del proceso de formación en ciencias, específicamente en elárea de la física eléctrica en el tema electrodinámica abordado por los estudiantes de secundaria de la institución educativa y con ello promover su participación activa en la comunidad.

En concordancia realizar un análisis profundo y concienzudo de los simuladores, permite que el docente planifique adecuadamente la estrategia pedagógica, el tipo de competencia y el nivel de construcción o estructuración de conocimiento se pretende desarrollar en el estudiante y potenciar eficientemente el uso de los simuladores(Ayala \& Salinas, 2019). Además, el identificar las particularidades, el tipo de construcción de las simulaciones. Si es una simulación deductiva, inductiva, de modelos causales cualitativos o de modelos semánticos, (Jonassen, 2004). Permite intuir como va a interactuar el estudiante con los elementos dispuestos en el simulador (componentes, variables, reacciones entre otros).

\section{REFERENTE TEÓRICO}

En las últimas décadas surge una variación al paradigma de la enseñanza-aprendizaje tradicional que abarca aspectos sobre el proceso de aprendizaje. Primero los seres humanos diariamente se enfrentan al desafío de producir conocimiento y no simplemente de reproducirlo, ya que la repetición no genera desafíos (S. Torres, 2005). Los estudiantes pueden alcanzar un nivel de competencia optimo cuando son motivados a involucrarse de activamente en el proceso de aprendizaje(Crawford, 2004).

De esta manera, según los avances en la psicología del aprendizaje, este deber ser activo, situado, contextual, interactivo, significativo y colaborativo, (Martinez, 2016). Se debe recordar la idea del cerebro funcionando en múltiples tareas. De tal modo, para generar un verdadero aprendizaje, debe entenderse que la mente procesa en paralelo múltiples tipos de información y estímulos simultáneamente (Sánchez, 2002). 
En virtud de estimular el aprendizaje se integra a la propuesta pedagógica el uso de simuladores por computadora. Cuya definición es amplia y variada. De este modo, el simulador es señalado como "un modelo científico de un sistema o proceso real o teórico que contiene información sobre el comportamiento del sistema el cual permite que haya experimentación" (Thomas \& Milligan, 2004), con la finalidad de aprender o evaluar estrategias para el funcionamiento del sistema (Shannon, 1988). Este entorno dinámico permite experimentar, lleva aproximaciones de una situación real de la teoría a la práctica, permitiendo al estudiante aprender manipulando y observando (Cunguan, 2019).

Un simulador es una mezcla de hardware y software en la que usando algoritmos se reproduce el comportamiento de un proceso, sistema o fenómeno físico, es decir que las condiciones reales son creadas artificialmente con el objeto de aprender, practicar acciones o habilidades que posteriormente pasan a una a un entorno real (Fiallos, 2012). Como es el caso de los pilotos aéreos, ellos practican en entornos simulados que asemejan las condiciones reales de un vuelo, les permiten practicar e incluso fallar para adquirir habilidades que posteriormente son llevadas a vuelos reales. Entonces, un simulador funciona como un sistema técnico que al imitar unas circunstancias reales, reproduce las condiciones propias de una actividad (Navarro, 2015).

Finalmente, los simuladores de calidad se caracterizan por contar con representaciones visuales de fenómenos naturales, modelos gráficos intuitivos, interactivos y con alto nivel de realismo, que apoyan la naturaleza activa del aprendizaje, interactividad con el conocimiento previo y el reacomodamiento de las nuevas estructuras cognitivas (adhieren nuevo conocimiento)(Velásquez, 2009). Su uso adecuado en determinados contextos del aprendizaje favorece el desarrollo conceptual en diversos campos (Velasco \& Buteler, 2017).
El descubrimiento de las formas de aprender la física se ha adaptado a lo largo de las últimas décadas. Se ha pasado de la trasmisión de información con tableros verdes, tiza y laboratorios físicos intocables para los estudiantes, a la gran cantidad de herramientas de material educativo computarizado que se encuentra disponible en la red y que usados adecuadamente potencializan un aprendizaje activo (Duart \& Sangrá, 2005). En este punto surgen las dudas de ¿cuál usar, como hacerlo, y cuál es la estrategia adecuada para integrarlo a un aula? Por lo anterior se realiza una revisión de los antecedentes investigativos asociados al uso de simuladores en el aprendizaje conceptual de la física dentro de una ventana de tiempo de 2011 a 2019 agrupados por aportes.

La mayoría de investigaciones destaca las bondades, aportes e impacto a nivel enseñanzaaprendizaje de la física con el uso de la simulación ( e.g.Albarracín \& Ramírez, 2017; Amaya, 2011; Benjumea, 2016; Cortes, 2016; Cunguan, 2019; Duran, 2019; Faúndez et al., 2017; Hernández et al., 2016; Lucero, 2015; Macías, 2018; Méndez, 2015; Morales, 2018; Peñata et al., 2019; Raviolo et al., 2011; Ruiz-Macías \& Duarte, 2018; Sanchez, 2018; Sanhueza et al., 2018; Á. Torres et al., 2017; Yánez, 2018). Sin embargo no se evidencian los aspectos o metodologias bajo los cuales se eligen la herramientas TIC a utilizar para garantizar una optima intervencion pedagogia.

\section{FÍSICA ELÉCTRICA EN LA EDUCACIÓN MEDIA}

La electricidad se deriva de la raíz griega elektron, que significa ámbar. "Electricidad se define como un fenómeno físico que se origina del movimiento de partículas subatómicas por medio de cargas eléctricas a través de la atracción y repulsión de las mismas" (Llamas, 2015). Es la fuente de energía básica que hace funcionar la tecnología que nos rodea; por lo que la física 
eléctrica en una rama importante de la básica al estudiar los fenómenos relacionados con las cargas eléctricas en reposo o movimiento.

De esta manera, los conceptos establecidos para impartir en física básica de secundaria en lo referente a física eléctrica deben abordar electricidad, con subtemas como electrostática y electrodinámica, además de magnetismo (incluyendo el tema electromagnetismo (Ministerio de Educación Nacional, 2006; Neira et al., 2018). Según las editoriales Santillana, McGraw-Hill y Norma para educación secundaria es básico abordar un desglose de temas como carga, campo, potencial y corriente eléctrica (Gutierrez, 2019; I. Morales \& Infante, 2005; Rojas \& Castaño, 2016) (Figura 1).

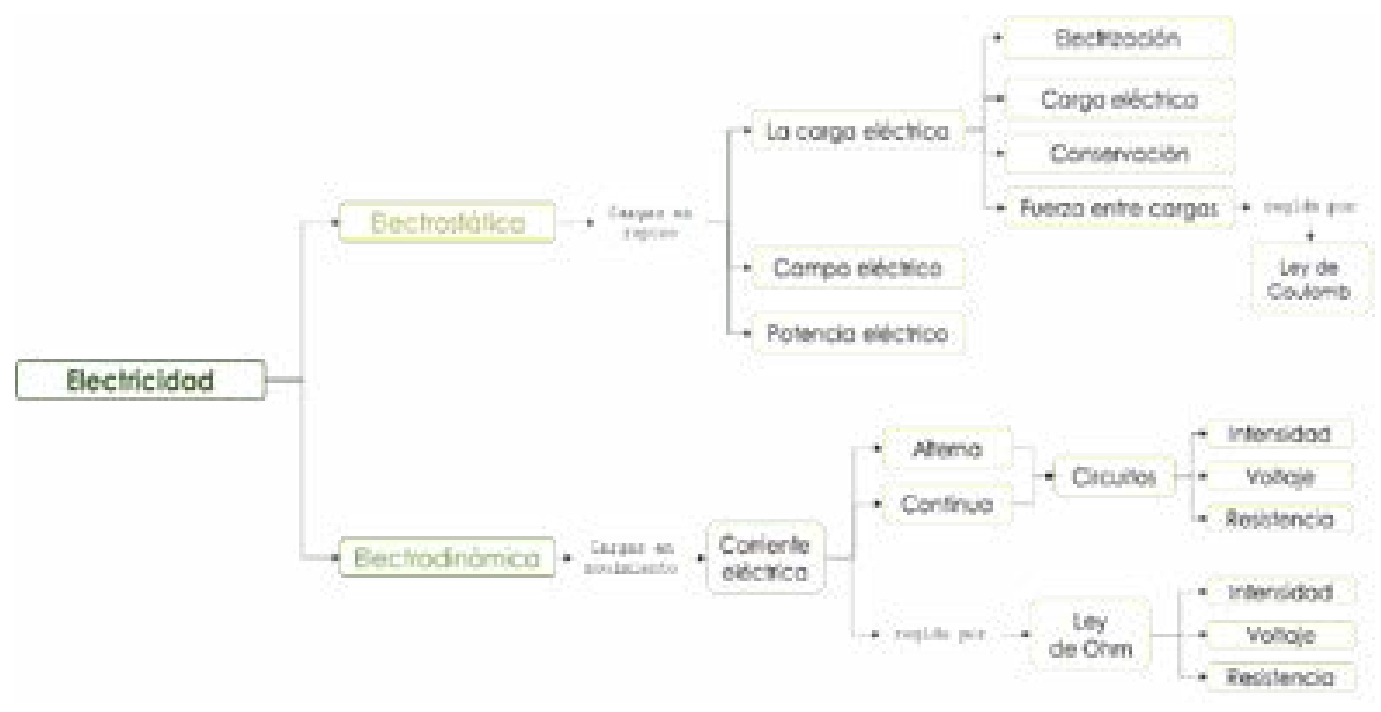

Figura 1. Ontología de la electricidad de la física eléctrica para la educación media

Fuente: Elaboración propia

\section{BENEFICIOSDEINTEGRARLOSSIMULADORES EN LAEDUCACIÓN}

Los simuladores, ha posibilitado múltiples efectos positivos en el proceso de enseñanza (Figura 2). En la experimentación, genera manipulación de las variables para probar hipótesis, realización de un número ilimitado y espontaneo de veces los experimentos en un entorno seguro y sin riesgo. Los estudiantes pueden inferir a través de la experimentación las características del modelo del fenómeno estudiado al comparar los resultados de una serie de experimentos. La repetición del mismo experimento bajo diferentes condiciones contribuye a la comprensión teórica de un fenómeno (Batanero, 2001). De igual manera, permiten al estudiante imitar el quehacer de un científico en la vida real, propiciando el desarrollo de habilidades científicas (Quevedo, 2013).

En el proceso de aprendizaje, permite al estudiante aprender a su ritmo, mejorar su comprensión y reflexión al cometer errores que cuestionan su aprendizaje, generan preguntas y guían la exploración dentro del simulador. Adicional, en el proceso de ensayo error (implícito en el uso de simuladores) se puede generar un proceso reflexivo de aprendizaje, orientado permanente por el docente(e.g. Huppert et al., 2002; Wieman et al., 2008).

En la enseñanza, faculta a los estudiantes la construcción de modelos representativos de un fenómeno y la posibilidad de comprobarlos Además, ayuda a fortalecer los conceptos, pues genera más posibilidad de practica; 
igualmente incrementa las oportunidades para generar relaciones causales entre el fenómeno de estudio y su significado(Amaya, 2011). Otra característica destacable es la facilitación de cambio conceptual que se genera cuando se aprende. La simulación muestra la naturaleza de un fenómeno, por lo que el estudiante puede libremente explorar, manipular y experimentar cambiando las condiciones y observando el resultado de forma inmediata, es de decir recibe un feedback inmediato (Thomas \& Milligan, 2004).

Teniendo en cuenta los aportes de (Amaya, 2011; Batanero, 2001; Cunguan, 2019; Fiallos, 2012; Huppert et al., 2002; Navarro, 2015; Quevedo, 2013; Thomas \& Milligan, 2004; Velasco \& Buteler, 2017; Velásquez, 2009;
Wieman et al., 2008) para esta investigación se define simulador como herramienta que intenta modelar aproximadamente el comportamiento de un fenómeno físico o entorno real, por medio de un programa por computadora., cuyas características deseadas son: la calidad gráfica, inductividad, interactividad, atractivo visualmente, fidelidad a la realidad del fenómeno, de fácil acceso, portabilidad, multiplataforma y seguro (no hay riesgo) para el usuario. El simulador debe permitir en relación al fenómeno simulado: observar, experimentar, explorar, manipular variables, repetir, probar hipótesis, inferir, comparar, reflexionar, generar preguntas, cometer errores, establecer relaciones causales y construir modelos representativos apoyados en el contexto del estudiante (Figura 2).

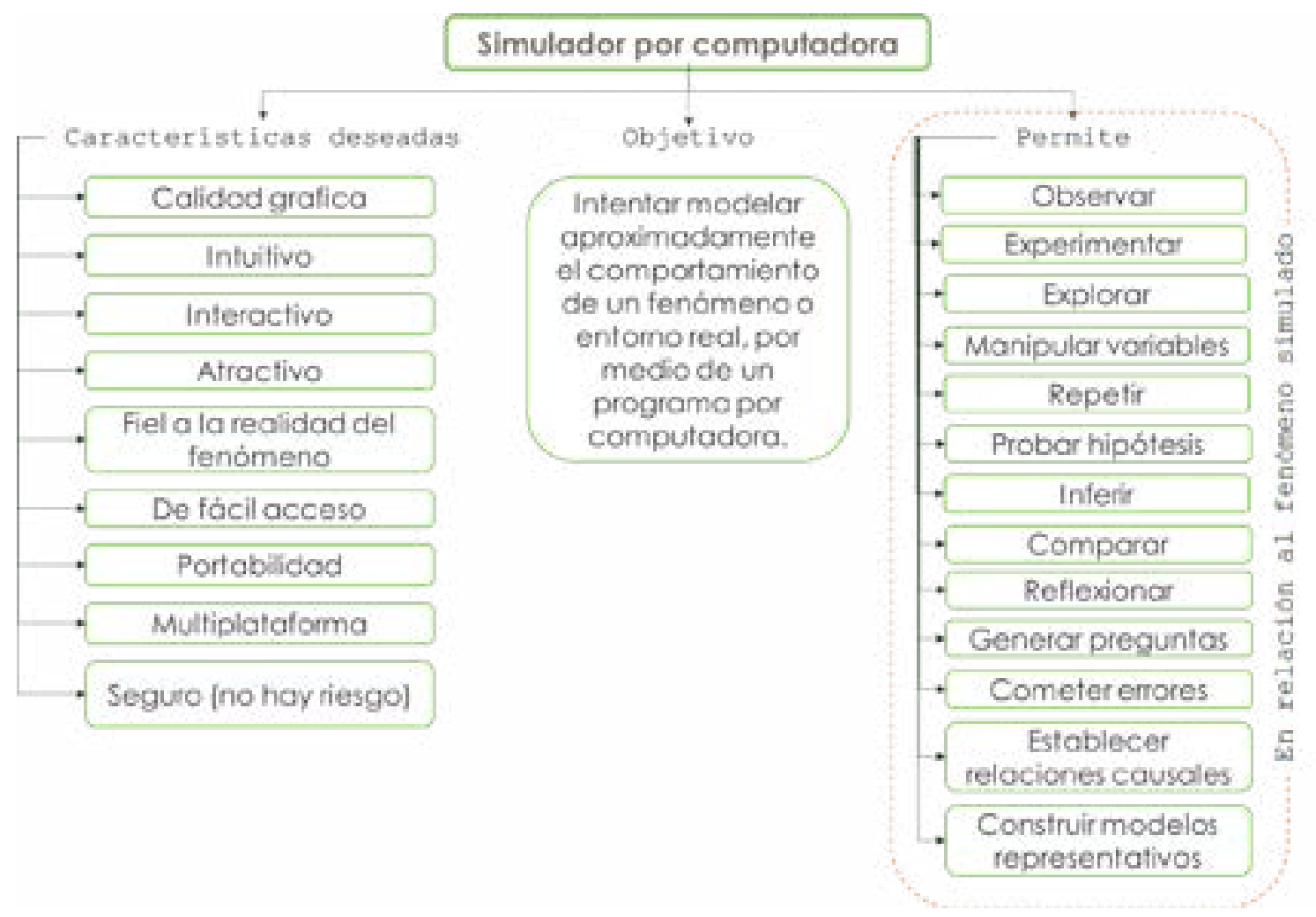

Figura 2. Características de un simulador.

Fuente: Autor. Basado en (Amaya, 2011; Batanero, 2001; Huppert et al., 2002; Quevedo, 2013;

Thomas \& Milligan, 2004; Wieman et al., 2008) 


\section{METODOLOGÍA}

Para llevar acabo la identificación y evaluación de los simuladores gráficos computacionales a ser usados como parte del diseño de una propuesta pedagógica. Se realiza el análisis descriptivo de la funcionalidad del software de simulación según la guía propuesta por Salinas y Ayala (2019) a dos simuladores disponibles en la web.

\section{INSTRUMENTO DE ANÁLISIS PARA SELECCIONAR SIMULADORES EDUCATIVOS}

Ayala y Salinas (2019) que afirman que la tercera forma de hacer ciencia se basa en los la modelación y la simulación ya que permite que el proceso de formación adquiera un nivel alto para ello se tienen en cuenta características de análisis funcional e instruccionales.

Como base se tienen en cuenta aspectos de análisis funcional que toma como referente: la identificación que establece la relación entre las características de los recursos, la funcionabilidad determina la competencia y nivel de estructuración, la fiabilidad define hasta qué punto logra emular la realidad, la confiabilidad posibilita la validación de los resultados, la usabilidad identifica la facilidad con la que se instala y usa el simulador, la eficiencia mide el rendimiento de la herramienta al abordar procesos complejos.

Identificar las características instruccionales se basa en: el mantenimiento que hace referencia a la facilidad de modificar o adaptar el simulador, la portabilidad que permite transferir datos del simulador entre entornos y la propuesta didáctica que establece las herramientas que apoyan el proceso educativo.

\section{POBLACIÓN}

Dentro de la identificación de los simuladores gráficos computacionales se establece como criterio de búsqueda únicamente los simuladores orientados a la enseñanza de la física eléctrica para educación media técnica.

En relación a la identificación se revisó la oferta de simuladores tanto en software libre como de pago que tengan propósito educativo y se enfoquen al abordaje de la enseñanza e la física eléctrica. Rodríguez-Hernández, A. (2010) realizo un estudio que identifico y catalogo simuladores en software para el aprendizaje y la enseñanza de la física, del cual se revisó los posibles recursos de software y se identificó el PhET Interactive Simulatios, proyecto de la Universidad de Colorado, disponible en varios idiomas, y siendo el simulador en software libre de mayor oportunidad de uso en ambientes de educación media.

Al no identificar otros simuladores en software libre para física electrica, se analizó software de pago, identificando Crocodile Physics Technology como una de las alternativas de mayor oferta en el mercado educativo.

\section{RESULTADOS Y DISCUSIÓN}

Los resultados de la identificación y análisis descriptivo de la funcionalidad del software de simulación se presentan conforme a los aspectos propuestos por Ayala y Salinas ( 2019) para dos simuladores PhET y Crocodile Physics - Technology que se presentan las tablas 1 a la tabla 8.

\section{IDENTIFICACIÓN}

Los simuladores comparados presentan similitudes en su descripción general, diferencias en el año de publicación y requerimientos técnicos (Ver Tabla 1). 
Tabla 1 Análisis comparativo en relación con la identificación de simuladores

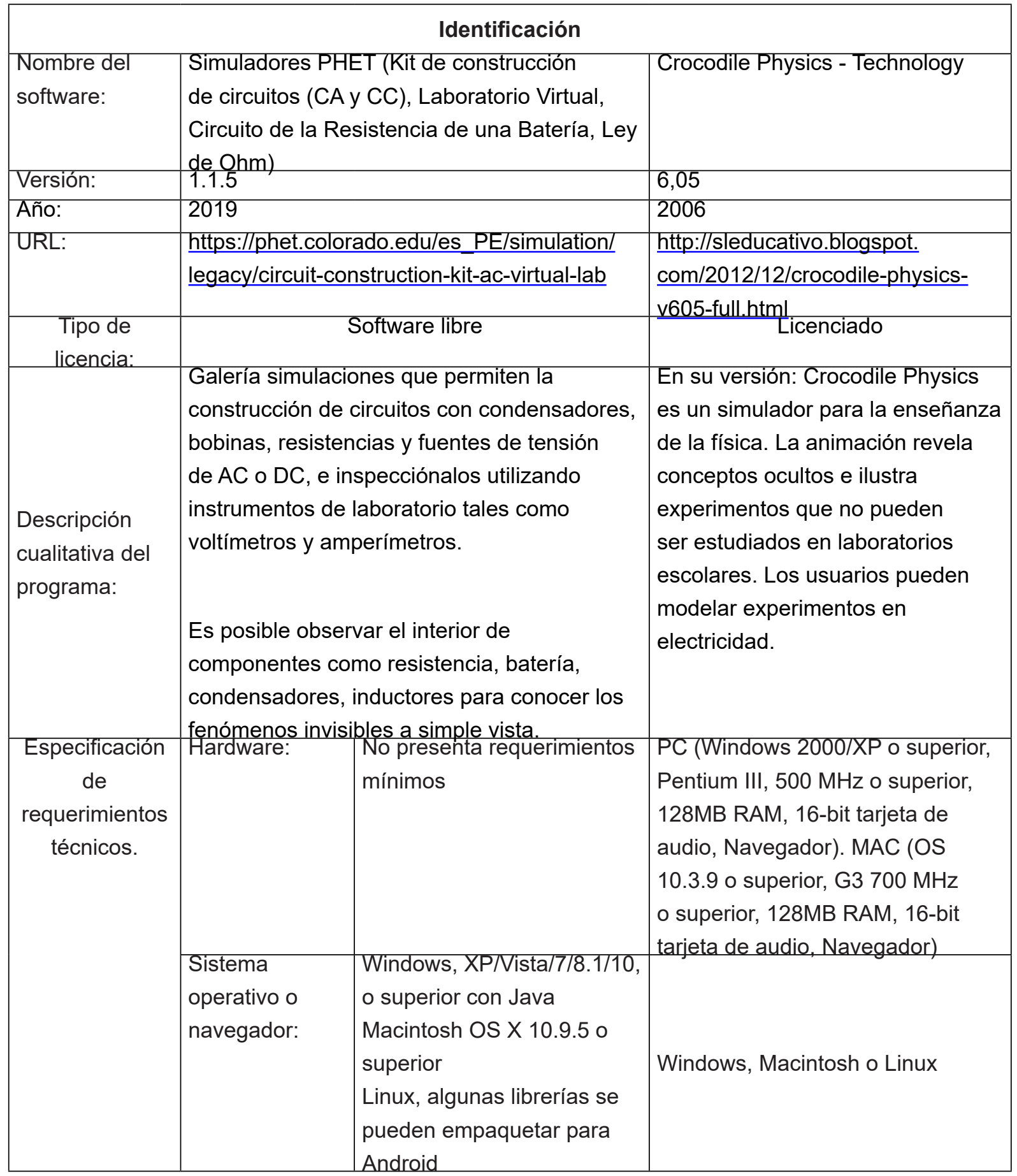

Fuente: Elaboración propia 


\section{FUNCIONALIDAD}

La Tabla 2 muestra la comparativa en términos de tipo de construcción del simulador, tipo de exploración del modelo simulado, fenómeno que se modela, interacción, resistencia a errores, recursividad, descripción de las variables y adecuación al currículo.

Tabla 2. Análisis comparativo en relación con la funcionalidad de simuladores

\begin{tabular}{|c|c|c|c|c|}
\hline & & Funcionabilidad & $\vdash$ & $\stackrel{0}{\bar{\Xi}}$ \\
\hline & & $\begin{array}{l}\text { Permite que el estudiante construya y compruebe } \\
\text { modelos de sistemas cerrados controlados por reacción. }\end{array}$ & $\mathbf{x}$ & $\mathbf{x}$ \\
\hline & $\begin{array}{l}\text { Construcción } \\
\text { de simulación } \\
\text { deductiva }\end{array}$ & $\begin{array}{l}\text { El modelo de simulación dinámica representa } \\
\text { conceptualmente la naturaleza cambiante de fenómenos } \\
\text { de sistemas de una forma similar al fenómeno real. }\end{array}$ & $\mathbf{x}$ & $\mathbf{x}$ \\
\hline & & $\begin{array}{l}\text { En este tipo de herramientas el modelo se concibe y se } \\
\text { implementa antes de ser comprobado. }\end{array}$ & $\mathbf{x}$ & $\mathbf{X}$ \\
\hline Tipo de & Construcción & $\begin{array}{l}\text { Permiten construir modelos dinámicos abiertos de } \\
\text { sistemas de fenómeno. }\end{array}$ & $\mathbf{x}$ & $\mathbf{x}$ \\
\hline $\begin{array}{l}\text { Construcción } \\
\text { de la } \\
\text { simulación: }\end{array}$ & $\begin{array}{l}\text { de simulación } \\
\text { inductiva }\end{array}$ & $\begin{array}{l}\text { Cada vez que se añade un elemento al sistema, se } \\
\text { puede comprobar el modelo para observar el efecto del } \\
\text { nuevo elemento en el funcionamiento del sistema. }\end{array}$ & $\mathbf{x}$ & $\mathbf{x}$ \\
\hline & $\begin{array}{l}\text { Construcción } \\
\text { de modelos } \\
\text { causales } \\
\text { cualitativos }\end{array}$ & $\begin{array}{l}\text { Permite construir sistemas expertos basados en } \\
\text { descripciones cualitativas de relaciones causales con } \\
\text { hechos y reglas si- entonces, para la toma de decisiones. }\end{array}$ & & \\
\hline & $\begin{array}{l}\text { Construcción } \\
\text { de modelos } \\
\text { semánticos }\end{array}$ & $\begin{array}{l}\text { Permite la representación de asociaciones semánticas } \\
\text { entre conceptos dominantes dentro de un campo de } \\
\text { conceptos. }\end{array}$ & & \\
\hline & & $\begin{array}{l}\text { La simulación está previamente construida para que los } \\
\text { estudiantes exploren y experimenten. }\end{array}$ & $\mathbf{x}$ & $x$ \\
\hline & De caja negra & No se explica el modelo implícito. & $\mathbf{x}$ & $\mathrm{X}$ \\
\hline Tipo de & & $\begin{array}{l}\text { Permite la manipulación de variables y la comprobación } \\
\text { de los resultados de estas. }\end{array}$ & $\mathbf{x}$ & $X$ \\
\hline Exploración & & $\begin{array}{l}\text { El sistema necesita que el estudiante construya el } \\
\text { modelo de forma explícita antes de comprobarlo. }\end{array}$ & & \\
\hline & $\begin{array}{l}\text { De caja de } \\
\text { cristal }\end{array}$ & $\begin{array}{l}\text { Se puede hacer seguimiento al comportamiento del } \\
\text { modelo subyacente en la simulación. }\end{array}$ & & \\
\hline & & $\begin{array}{l}\text { Permite la manipulación de variables y la comprobación } \\
\text { de los resultados de estas. }\end{array}$ & & \\
\hline
\end{tabular}




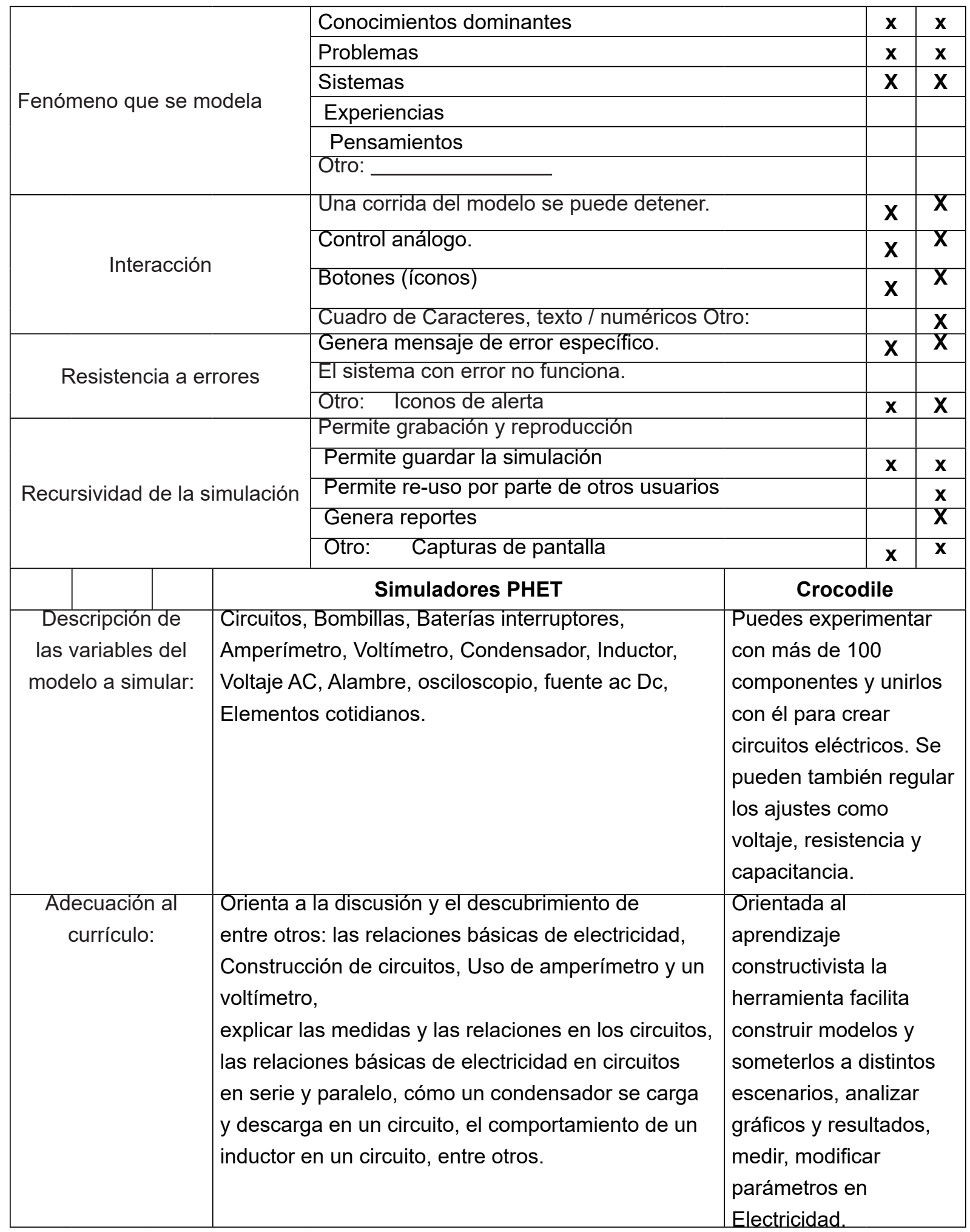

Fuente: Elaboración propia 


\section{CONFIABILIDAD /FIABILIDAD}

En la Tabla 3 se comparan aspectos de confiabilidad / fiabilidad en cuanto los sistemas de representación, nivel de abstracción, profundización, respaldo y validación de los simuladores PHET y Crocodile.

Tabla 3. Análisis comparativo en relación con la Confiabilidad /fiabilidad de simuladores

\begin{tabular}{|c|c|c|c|c|}
\hline \multicolumn{5}{|c|}{ Confiabilidad / fiabilidad } \\
\hline \multirow{20}{*}{$\begin{array}{l}\text { Sistema de } \\
\text { representación }\end{array}$} & \multicolumn{4}{|c|}{ Los elementos del modelo o fenómeno a simular se representan con } \\
\hline & & PHET & Crocodile \\
\hline & \multicolumn{2}{|l|}{ Tconos. } & $\mathrm{X}$ & $\mathrm{X}$ \\
\hline & \multicolumn{2}{|l|}{ Imágenes planas. } & $\mathbf{X}$ & $\mathrm{X}$ \\
\hline & \multicolumn{2}{|l|}{ Tmágenes 3D de baja definición. } & $\mathbf{X}$ & $\mathbf{X}$ \\
\hline & \multicolumn{2}{|l|}{ Imágenes 3D de alta definición } & & \\
\hline & \multicolumn{2}{|l|}{ Realidad aumentada } & & \\
\hline & \multicolumn{2}{|c|}{ Otro: } & & \\
\hline & \multicolumn{4}{|c|}{ Los cambios en el modelo o fenómeno a simular se representan con: } \\
\hline & \multicolumn{2}{|c|}{ Datos numéricos o texto. } & $\mathrm{X}$ & $\mathbf{x}$ \\
\hline & \multicolumn{2}{|l|}{ Cambio de imágenes 2D. } & $\mathrm{X}$ & $\mathbf{X}$ \\
\hline & \multicolumn{2}{|l|}{ Movimiento animado. } & $\mathbf{x}$ & $\mathbf{X}$ \\
\hline & \multicolumn{2}{|l|}{ Otro: } & & \\
\hline & \multicolumn{4}{|c|}{$\begin{array}{l}\text { El escenario donde se encuentra el modelo o fenómeno a simular se representan } \\
\text { con: }\end{array}$} \\
\hline & \multicolumn{2}{|c|}{ Fondo unicolor. } & $\mathbf{X}$ & $\mathbf{X}$ \\
\hline & \multicolumn{2}{|l|}{ Imagen plana estática. } & X & $\mathbf{X}$ \\
\hline & \multicolumn{2}{|l|}{ Imagen plana en movimiento. } & & \\
\hline & \multicolumn{2}{|l|}{ Imagen 3D estática. } & & \\
\hline & \multicolumn{2}{|l|}{ Animación 3D con movimiento. } & & \\
\hline & \multicolumn{2}{|l|}{ Otro: Animación plana } & X & \\
\hline \multirow[b]{2}{*}{$\begin{array}{l}\text { Descripción } \\
\text { del nivel de } \\
\text { Abstracción de la } \\
\text { simulación: }\end{array}$} & \begin{tabular}{l|l} 
& Simuladores PHET \\
\end{tabular} & \multicolumn{3}{|c|}{ Crocodile Physics - Technology } \\
\hline & $\begin{array}{l}\text { Presenta elementos contextualizados a } \\
\text { la realidad e iconografía técnica }\end{array}$ & \multicolumn{3}{|c|}{$\begin{array}{l}\text { Presenta elementos } \\
\text { contextualizados a la realidad e } \\
\text { iconografía técnica dependiendo el }\end{array}$} \\
\hline $\begin{array}{l}\text { Descripción } \\
\text { del nivel de } \\
\text { profundización de } \\
\text { la simulación: }\end{array}$ & $\begin{array}{l}\text { permite de forma gráfica observar el } \\
\text { movimiento de electrones, formas de } \\
\text { onda, comportamiento de elemento, } \\
\text { reacción en cadena de los fallos, valores } \\
\text { de los elementos de forma dinámica }\end{array}$ & \multicolumn{3}{|c|}{$\begin{array}{l}\text { permite formas de onda, } \\
\text { comportamiento de elemento, } \\
\text { reacción en cadena de los fallos, } \\
\text { valores de los elementos de forma } \\
\text { dinámica }\end{array}$} \\
\hline
\end{tabular}




\begin{tabular}{|c|c|c|}
\hline $\begin{array}{l}\text { Respaldo del } \\
\text { software: }\end{array}$ & $\begin{array}{l}\text { Fundado por Carl Wieman Premio } \\
\text { Nobel de Física por su investigación } \\
\text { experimental en física atómica. } \\
\text { PhET interactivo simulación. } \\
\text { Desarrollado por la Universiada de } \\
\text { colorado. Patrocinado por empresas } \\
\text { como: Google.org, } \\
\text { The William and Flora Hewlett Fundación } \\
\text { JILA, ERCSME at King Saud University, } \\
\text { Moore Foundation, } \\
\text { New Schools Venture Fund, The National } \\
\text { Science Foundation, The O'Donnell } \\
\text { Foundation, } \\
\text { C. Wieman and S. Gilbert, King Saud } \\
\text { University, The Kavli Operating Institute }\end{array}$ & $\begin{array}{l}\text { Modelos diseñados por Sumdog } \\
\text { Ltd (Crocodile Clips). }\end{array}$ \\
\hline $\begin{array}{l}\text { Descripción de } \\
\text { la validación de } \\
\text { los resultados de } \\
\text { simulación: }\end{array}$ & $\begin{array}{l}\text { Los principios de diseño de simulación } \\
\text { PhET se basan en la investigación } \\
\text { sobre cómo aprenden los estudiantes } \\
\text { (Bransford et al., 2000)y de las } \\
\text { entrevistas de simulación. Entre cuatro } \\
\text { y seis entrevistas de estilo Piensa en } \\
\text { Voz Alta se realizan con estudiantes } \\
\text { individuales con cada simulación. Estas } \\
\text { entrevistas son una fuente rica de datos } \\
\text { para el estudio de diseño de la interfaz } \\
\text { y el aprendizaje del estudiante. El PhET } \\
\text { Look and Fiel describe brevemente los } \\
\text { principios de diseño de interfaz y una } \\
\text { discusión completa se encuentra en } \\
\text { varios artículos por Adams et al., (2008) }\end{array}$ & $\begin{array}{l}\text { No se evidencia por parte los } \\
\text { creadores las pruebas hechas } \\
\text { para validar los resultados de la } \\
\text { simulación. }\end{array}$ \\
\hline
\end{tabular}

Fuente: Elaboración propia

\section{USABILIDAD}

La Tabla 4 muestra la evaluación en términos de usabilidad en aspectos como la facilidad de instalación, disponibilidad de manual de usuario, entrenamiento, facilidad de uso e identificación de errores del simulador y si posible conectar sus resultados a otros softwares. 
Tabla 4. Análisis comparativo en relación con la usabilidad de simuladores

\begin{tabular}{|c|c|c|}
\hline \multicolumn{3}{|c|}{ Usabilidad } \\
\hline & PHET & Crocodile \\
\hline $\begin{array}{l}\text { Facilidad de la } \\
\text { instalación: }\end{array}$ & No requiere instalación. & $\begin{array}{l}\text { Instalación sencilla tiempo estimado } 2 \\
\text { minutos }\end{array}$ \\
\hline $\begin{array}{l}\text { Manual de } \\
\text { usuario: }\end{array}$ & $\begin{array}{l}\text { No se presenta manual de usuario oficial. } \\
\text { Entorno presenta mensajes emergentes } \\
\text { de ayuda. }\end{array}$ & $\begin{array}{l}\text { Presenta pestaña de ayuda y manual } \\
\text { en idioma inglés, y soporte técnico al } \\
\text { usar licencias de pago. }\end{array}$ \\
\hline Entrenamiento: & $\begin{array}{l}\text { No requiere entrenamiento previo } \\
\text { ya que presenta un menú básico de } \\
\text { componentes y opciones en un solo } \\
\text { tablero. }\end{array}$ & $\begin{array}{l}\text { Por su mediana complejidad requiere } \\
\text { tiempo de entrenamiento con espacio } \\
\text { de trabajo librerías. }\end{array}$ \\
\hline $\begin{array}{l}\text { Facilidad de } \\
\text { uso: }\end{array}$ & $\begin{array}{l}\text { El entorno es intuitivo, iconos y lenguaje } \\
\text { son habituales, el software presenta una } \\
\text { complejidad de uso media. }\end{array}$ & $\begin{array}{l}\text { el entorno es intuitivo, iconos y lenguaje } \\
\text { son habituales, el software presenta } \\
\text { una complejidad alta para estudiantes } \\
\text { de educación media sin experiencia en } \\
\text { uso de simuladores, }\end{array}$ \\
\hline $\begin{array}{l}\text { Aporte a } \\
\text { errores de uso: }\end{array}$ & $\begin{array}{l}\text { Indica los posibles errores mediante } \\
\text { ménades de alerta o gráficos de } \\
\text { comportamiento. }\end{array}$ & $\begin{array}{l}\text { Indica los posibles errores mediante } \\
\text { ménades de alerta o gráficos de } \\
\text { comportamiento. }\end{array}$ \\
\hline $\begin{array}{l}\text { Posibilita la } \\
\text { conexión de } \\
\text { redes y bases } \\
\text { de datos: }\end{array}$ & $\begin{array}{l}\text { No permite extraer datos o bases de } \\
\text { datos ni guarda perfiles de la simulación. }\end{array}$ & $\begin{array}{l}\text { Permitente exportar e importar archivos } \\
\text { de simulación, bases de datos de las } \\
\text { respuestas de los componentes o } \\
\text { sistema simulado. }\end{array}$ \\
\hline
\end{tabular}

Fuente: Elaboración propia

\section{EFICIENCIA}

La Tabla 5 presenta los resultados comparativos frente a la eficiencia de los simuladores frente al rendimiento, comportamiento en el tiempo y uso de recursos. 
Tabla 5. Análisis comparativo en relación con la eficiencia de simuladores

\begin{tabular}{|l|l|l|}
\hline \multicolumn{2}{|c|}{ Eficiencia } \\
\hline & \multicolumn{1}{|c|}{ PHET } & \multicolumn{1}{|c|}{ Crocodile } \\
\hline Rendimiento: & $\begin{array}{l}\text { Presenta rendimiento óptimo en relación } \\
\text { a capacidad de maquina y exigencia de } \\
\text { la simulación. }\end{array}$ & $\begin{array}{l}\text { Presenta rendimiento óptimo en } \\
\text { relación a capacidad de maquina y } \\
\text { exigencia de la simulación. }\end{array}$ \\
\hline $\begin{array}{l}\text { Comportamiento } \\
\text { con el tiempo: }\end{array}$ & $\begin{array}{l}\text { es posible variar la velocidad de } \\
\text { animación, indica cuando los valores } \\
\text { exceden la capacidad de proceso } \\
\text { Requerimiento básico ligado a } \\
\text { navegador de internet o instalación de } \\
\text { recursos: }\end{array}$ & $\begin{array}{l}\text { Es posible variar la velocidad de } \\
\text { animación, indica cuando los valores } \\
\text { exceden la capacidad de proceso } \\
\text { Java }\end{array}$ \\
\hline
\end{tabular}

Fuente: Elaboración propia

\section{MANTENIMIENTO}

La Tabla 6 muestra la comparativa frente al mantenimiento de los simuladores para lo cual no se evidencio manuales de mantenimiento.

Tabla 6. Comparativa en elemento mantenimiento de simuladores

\begin{tabular}{|l|c|}
\hline \multicolumn{2}{|c|}{ Mantenimiento } \\
\hline \multicolumn{1}{|c|}{ PHET } & Crocodile \\
\hline $\begin{array}{l}\text { No se evidencia manual de mantenimiento, pero } \\
\text { es posible apostar en el desarrollo o mejora de la } \\
\text { herramienta. }\end{array}$ & $\begin{array}{c}\text { No se evidencia manual de } \\
\text { mantenimiento. }\end{array}$ \\
\hline
\end{tabular}

Fuente: Elaboración propia

\section{PORTABILIDAD}

La Tabla 7 presenta las características de portabilidad de PHET y Crocodile.

Tabla 7. Comparativa en elemento portabilidad de simuladores

\begin{tabular}{|l|c|c|}
\hline \multicolumn{2}{|c|}{ PHET } & Crocodabilidad \\
\hline & & \\
\hline Posibilidad & Es fácilmente trasferible en entornos con & Es fácilmente trasferible en entornos con \\
de & sistemas operativos Windows, Macintosh y & sistemas operativos Windows, Macintosh \\
transferir el & Linux; También es posible empaquetar las & y Linux. \\
software de & versiones htm5 en aplicación para Android. & \\
un entorno & & \\
a otro: & & \\
\hline
\end{tabular}




\begin{tabular}{|l|c|c|}
\hline $\begin{array}{l}\text { Intercambio } \\
\text { de datos: }\end{array}$ & $\begin{array}{c}\text { Permite guardar esquemáticos de los } \\
\text { circuitos simulados. Y ser usados con otras } \\
\text { simulaciones de PHET. }\end{array}$ & $\begin{array}{c}\text { Permite guardar esquemáticos de los } \\
\text { circuitos simulados. Y ser usados con } \\
\text { otras simulaciones. }\end{array}$ \\
\hline
\end{tabular}

Fuente: Elaboración propia

\section{PROPUESTA DIDÁCTICA}

Tabla 8. muestra la estrategias o propuestas didácticas brindadas por los simuladores y disponibles para integrar su uso en el aula.

Tabla 8. Comparativa en elemento propuesta didáctica de simuladores

\begin{tabular}{|l|c|}
\hline \multicolumn{2}{|c|}{ Propuesta didáctica } \\
\hline \multicolumn{1}{|c|}{ PHET } & Crocodile \\
\hline $\begin{array}{l}\text { Pone a disposición de los usuarios el procedimiento de uso libre para creación } \\
\text { de actividades con las simulaciones interactivas bajo enfoque de indagación } \\
\text { guiada. }\end{array}$ & No se evidencia. \\
\hline
\end{tabular}

Fuente: Elaboración propia

\section{CONCLUSIONES}

Los simuladores gráficos computacionales identificados presentan diferencia significativa en el año de publicación y Crocodile tiene desventaja ya no es posible instalarlo en dispositivos con sistema operativo Android. Así mismo PHET presenta ventaja al ser un software de uso libre de a tabla 1.

En términos de funcionalidad los simuladores PHET y Crocodile presenta similitudes en el tipo de construcción de la simulación evaluados desde la perspectiva propuesta por Jonassen, (2004) , al presentar una construcción con aspectos de simulación deductiva e inductiva según el módulo o librería que se use. Adicional presentan un tipo de exploración del modelo simulado de caja negra ya que la simulación esta previamente construida y no se explica el modelo implícito.

Frente a la comparativa de confiabilidad/fiabilidad de los simuladores PHET y Crocodile, presentan similitud en el sistema de representación y nivel de abstracción. Pero PHET establece un nivel de profundización grafico ajustado a educación media; esta característica puede ser aprovecha en la implementación de la propuesta pedagógica ya que, al reconocer el tipo de sistema simbólico, el docente planifica a intensión el uso del simulador en el proceso de aprendizaje teniendo en cuenta que las personas usan los sistemas simbólicos para regular sus procesos mentales y la actividad de las otras personas mediante la interacción y comunicación (Coll, 2004).

Se resalta que los simuladores PHET a presenta respaldo de un equipo interdisciplinario de la universidad del colorado y más de 50 organizaciones internacionales que patrocinan su desarrollo(Colorado, 2020), frente a Crocodile desarrollado por la empresa Sumdog Ltd.

En cuanto a la usabilidad de Crocodile presenta manuales de usuario, posibilidad de exportar datos y por su complejidad requiere entrenamiento previo a su uso para estudiantes de ecuación media. En cuanto a su entono ambos son intuitivos y presentan sistemas de alerta en mensajes e iconos. 
Respecto a la eficiencia los simuladores presentan características similares, pero para su uso Crocodile consume mayores recursos de máquina. En temas de mantenimiento los simuladores no evidencian requerimientos. Como característica de portabilidad ambos pueden ser ejecutados en sistemas operativos Windows, Macintosh y Linux, pero únicamente PHET puede ser usado desde Android en su versión html5.

Aplicada la guía de Ayala y Salinas (2019) para el análisis descriptivo de la funcionalidad de los simuladores PHET y Crocodile, se establece que por sus características los simuladores PHET presentan un mayor grado de adaptabilidad al contexto, aporte al cumplimiento de los objetivos de aprendizaje y competencias a desarrollar dentro del proyecto de investigación "aprendizaje de conceptos en física eléctrica integrando simuladores".

\section{REFERENCIAS BIBLIOGRÁFICAS}

Adams, W. K., Reid, S., Lemaster, R., McKagan, S. B., Perkins, K. K., Dubson, M., \& Wieman, C. E. (2008). A study of educational simulations Part 1 - Engagement and learning. Journal of Interactive Learning Research, 19(3), 397-419.

Albarracín, R., \& Ramírez, M. (2017). Aplicación del sistema 4MAT apoyado en las simulaciones PhET para el desarrollo de competencias científicas empleando como eje de aprendizaje el tema de ondas. LatinAmerican Journal of Physics Education, 11(3), 8. http://www.lajpe.org

Amaya, G. (2011). La simulación computarizada como instrumento del método en el proceso de enseñanza y aprendizaje de la física, desde la cognición situada: ley de OHM. Actualidades Investigativas en Educación, 8(1). https://doi.org/10.15517/ aie.v8i1.9321
Ayala, J., \& Salinas, J. (2019). Instrumento de análisis para seleccionar simuladores educativos. XXII Congreso internacional tecnología e innovación para la diversidad de los aprendizajes EDUTEC. https://www. researchgate.net/publication/340633383_ Instrumento_de_analisis_para_ seleccionar_simuladores_educativos

Batanero, C. (2001). Aleatoriedad, Modelización, Simulación. http://www.ugr.es/ batanero/ pages/ARTICULOS/Jaem2001.pdf

Benjumea, A. L. (2016). La Simulación, una herramienta para el aprendizaje de los conceptos físicos. Tesis, 91.

Bransford, J. D., Brown, A. L., Suzanne Donovan, M., \& Pellegrino, J. W. (2000). How People Learn. http://www.nap.edu

Coll, C. (2004). Psicología de la educación y prácticas educativas mediadas por las tecnologías de la información y la comunicación: una mirada constructivista. Sinéctica, 25, 1-24. http://biblio.uoc. es:443/docs_elec/2704.htm

Colorado, U. de. (2013). Física - Simulaciones PhET. https://phet.colorado.edu/es/ simulations/category/physics

Cortes, A. B. (2016). Desarrollo de un entorno de simulación $3 D$ como herramienta de apoyo para la enseñanza de la física Universidad de montemorelos facultad de ingeniería y tecnología.

Crawford, M. L. (2004). Enseñanza Contextual. Investigación, Fundamentos y Técnicas para Mejorar la Motivación y el Logro de los Estudiantes en Matemática y Ciencias. www.cord.org

Cunguan, M. L. (2019). Modelización de las Leyes de Newton en el cuerpo humano y la enseñanza interactiva mediante el uso 
del simulador PhET en los estudiantes de primero de bachillerato técnico en mecánica automotriz (EMA) de la Unidad Educativa Rumiñahui ubicada en la ciudad d. Quito: UCE.

Duart, J. M., \& Sangrá, A. (2005). Formación universitaria por medio de la web: un modelo integrador para el aprendizaje superior (23-49), Aprender a lo largo de la vida y las nuevas tecnologías (51-60), Educar en valores por medio de la web (61-75), La motivación como interacción entre. Aprender En La Virtualidad.

Duran, D. (2019). Actitud de los estudiantes hacia la física con la implementación del uso de simuladores de fenómenos físicos en clases.

Faúndez, C. A., Bravo, A. A., Ramírez, G. P., \& Astudillo, H. F. (2017). Tecnologías de la Información y la Comunicación (TIC) en el proceso de enseñanza-aprendizaje de conceptos de termodinámica como herramienta para futuros docentes. Formacion Universitaria, 10(4), 4354. $\quad$ https://doi.org/10.4067/S071850062017000400005

Fiallos, C. (2012). Los simuladores educativos. http://cinthyaraquelfiallos.blogspot. com/2012/04/simuladores.html

Gutierrez, C. (2019). FISICA /I (MC GRAW HILL (ed.)).

Hernández, J. E., Timóteo, D. J. A., \& Kuleshov, V. (2016). PhET: Alternative and effective tool in the teaching of the topic "Alternating Current"; PhET: Herramienta alternativa y eficaz en la enseñanza del tema "Corriente Alterna." Proceedings 4to Coloquio Internacional CIFAC, 11 pages.
Huppert, J., Lomask, S., \& Lazarowitz, R. (2002). Computer simulations in the high school: Students' cognitive stages, science process skills and academic achievement in microbiology. International Journal of Science Education, 24(8), 803-821. https:// doi.org/10.1080/09500690110049150

Jonassen, D. (2004). Del Docente Presencial Al Docente Virtual. https://www.slideshare. net/marlonint45/del-docente-presencialal-docente-virtual

Llamas, L. (2015). Física II. Secretaría de Educación Pública. https://www.dgb. sep.gob.mx/servicios-educativos/ telebachillerato/LIBROS/4-semestre-2016/ Fisica-II.pdf

Lucero, I. (2015). Resolviendo problemas de Física con simulaciones: un ejemplo para el ciclo básico de la educación secundaria. $X$ Congreso de Tecnología En Educación \& Educación En Tecnología, 645-653. http:// sedici.unlp.edu.ar/handle/10915/49071

Macías, E. R. (2018). Desarrollo de una estrategia didáctica mediada por MEC para el estudio de oscilaciones y ondas. https://repositorio.uptc.edu.co/ handle/001/2329

Martinez, J. E. (2016). PhET. Percepciones y contribucion del uso de simulaciones en el aprendizaje de los conceptos de energia para un curso de fisica general de la ensenanza tecnica. http://arxiv.org/ abs/1606.00245

Méndez, D. (2015). Estudio de las motivaciones de los estudiantes de secundaria de física y química y la influencia de las metodologías de enseñanza en su interés. Educacion $X X 1,18(2), 215-235$. https:// doi.org/10.5944/educXX1.14016 
Ministerio de Educación Nacional. (2006). Estándares Básicos de Competencias en lenguaje, matematicas, ciencias y ciudadanas. Revolución Educativa, 3, 1-184. https://www.mineducacion. gov.co/1759/w3-article-340021.html? noredirect $=1$

Morales, G. N. (2018). Simulación computacional del concepto de gradiente alternante: una propuesta para el acercamiento a la física de aceleradores.Trabajo de grado http://repository.pedagogica.edu.co/ handle/20.500.12209/11273

Morales, I., \& Infante, E. (2005). Física 2 (Norma (ed.)).

Navarro, J. (2015). Definición de Simulador. Definición ABC. https://www.definicionabc. com/tecnologia/simulador.php

Neira, A., Ochoa, O., Avendaño, O., \& Vacca, J. (2018). Plan de área ciencias naturales y educación ambiental - Institución Educativa Enrique Suarez - Almeida (p. 50).

Nieda, J., \& Macedo, B. (1997). Importancia de la enseñanza de las ciencias en la sociedad actual. In Un Curriculo Científico Para Estudiantes De 11 a 14 Años (pp. 19-24). https://www.oei.es/historico/oeivirt/curricie/ curri01.htm

Peñata, A. E., Camargo, E. A., \& Garcia, L. F. (2019). Implementación de simulaciones virtuales en la enseñanza de física y química para la educación media en la subregión de Urabá, Antioquia. Journal of Chemical Information and Modeling, 53(9), 1689-1699. https://doi.org/10.1017/ СBO9781107415324.004

Quevedo, L. (2013). Diseño instruccional en simuladores de física. https://reposital. cuaed.unam.mx:8443/xmlui/bitstream/ handle/123456789/4413/Lidice Quevedo_
Diseño Instruccional en simuladores de física. pdf?sequence=1\&isAllowed=y

Raviolo, A., Alvarez, M., \& Aguilar, A. (2011). La hoja de cálculo en la enseñanza de la Física: re-creando simulaciones. Revista de Enseñanza de La Física, 24(1), 97-107. http://rid.unrn.edu.ar/jspui/ handle/20.500.12049/2356

Rodríguez-Hernández, A. (2010). Definición, descripción y estudio de los simuladores en SOFTWARE libre utilizados para el aprendizaje de la física. Revista De Investigaciones UNAD, 9(1), 153-169. https://doi.org/10.22490/25391887.657

Rojas, D., \& Castaño, J. (2016). Proyecto saberes física 11 (Santillana (ed.)).

Ruiz-Macías, E., \& Duarte, J. E. (2018). Diseño de un material didáctico computarizado para la enseñanza de Oscilaciones y Ondas, a partir del estilo de aprendizaje de los estudiantes. Revista de investigación, desarrollo e innovación, 8(2), 295. https://doi.org/10.19053/20278306. v8.n2.2018.7966

Sanchez, N. (2018). Aprendizaje del concepto de trabajo mecánico con simulaciones virtuales a traves de una secuencia didáctica. http://repositorio.unicauca.edu. co:8080/handle/123456789/1151

Sanhueza, S., Bravo, A., Faúndez, C., \& Utreras, E. (2018). Las TIC como herramientas cognitivas de inclusión en clases de física para estudiantes de enseñanza secundaria. Góndola, Enseñanza y Aprendizaje de Las Ciencias, 13(2), 306-324. https://doi. org/10.14483/23464712.12585

Shannon, R. (1988). Simulación de Sistemas. Diseño, desarrollo e implementación. 
Thomas, R. C., \& Milligan, C. D. (2004). Putting Teachers in the Loop: Tools for Creating and Customising Simulations. Journal of Interactive Media in Education, 2004(2), 24. https://doi.org/10.5334/2004-15

Torres, Á., Bañón, D., \& López, V. (2017). Empleo de smartphones y apps en la enseñanza de la física y química. Enseñanza de Las Ciencias: Revista de Investigación y Experiencias Didácticas, Extra, 671. https://www.raco.cat/index.php/ Ensenanza/article/view/334743

Torres, S. (2005). Propuesta metodológica de enseñanza y aprendizaje de la geometría, aplicada en escuelas críticas. http:// repositorio.uchile.cl/tesis/uchile/2005/ lastra_s/sources/lastra_s.pdf

UNESCO. (1999). Declaración sobre la ciencia y la utilización del conocimiento científico. (Conferencia Mundial sobre la Ciencia). Acta de La Conferencia Mundial Sobre Las Ciencias Del Primero de Julio de 1999., 1-8. https://repositorio.uam.es/ bitstream/handle/10486/684760/EM_2_8. pdf?sequence $=1$

Velasco, J., \& Buteler, L. (2017). Computational simulations in physics education: A critical review of the literature. In Ensenanza de las Ciencias (Vol. 35, Issue 2, pp. 161-178). Universitat Autònoma de Barcelona. https:// doi.org/10.5565/rev/ensciencias. 2117

Velásquez, Á. (2009). Importancia de la instrumentación y la simulación de fenómenos naturales dentro de la formación en ciencias aplicadas (p. 32).

Wieman, C., Adams, W., \& Perkins, K. (2008). Physics. PhET: Simulations that enhance learning. In Science (Vol. 322, Issue 5902, pp. 682-683). https://doi.org/10.1126/ science.1161948
Yánez, A. F. (2018). Simulador PhET en la enseñanza de las cargas eléctricas en movimiento en los estudiantes de tercero de bachillerato de la Unidad Educativa "Paúl Dirac", durante el año lectivo 20172018. http://www.dspace.uce.edu.ec/ bitstream/25000/15336/1/T-UCE-0010MF028-2018.pdf\%0Ahttp://www.dspace. uce.edu.ec/handle/25000/15336 